\title{
Prognostic models for alcoholic hepatitis
}

\author{
Erik Rahimi ${ }^{*}$ and Jen-Jung Pan
}

\begin{abstract}
Alcoholic hepatitis $(\mathrm{AH})$ is caused by acute inflammation of the liver in patients that consume excessive amounts of alcohol, usually in a background of cirrhosis. AH can range from mild to severe, life threatening disease with a high rate of short and long-term mortality. Prognostic models have been used to estimate mortality in order to identify those that may benefit from corticosteroids or pentoxifylline. This review focuses on the different prognostic models proposed. While limitations of the prognostic models exist, combining models may be beneficial in order to identify responders to therapy versus non-responders.
\end{abstract}

Keywords: Alcoholic hepatitis, Prognostic score, Mortality, Risk

\section{Introduction}

Alcoholic hepatitis $(\mathrm{AH})$ is a syndrome due to acute inflammation of the liver in patients that consume excessive amounts of alcohol [1]. Rapid onset of jaundice due to parenchymal injury ranging from mild to severe, lifethreatening disease usually in the background of concomitant cirrhosis is the hallmark presentation of $\mathrm{AH}$ [2]. Along with jaundice, varying symptoms and signs consist of fever, encephalopathy, abdominal distress, ascites, varices, anemia, leukocytosis, coagulopathy, and a ratio of serum aspartate aminotransferase (AST) to alanine aminotransferase (ALT) greater than 2, which rarely exceeds $300 \mathrm{IU}$ per liter, are seen [3-5]. Although it is a treatable form of alcoholic liver disease, there is a high rate of short and long-term mortality. The overall inpatient mortality was $6.6 \%-6.8 \%$ for acute $\mathrm{AH}$ and $13.6 \%$ for chronic $\mathrm{AH}$ in population-based studies $[1,6]$. In a Danish study, the 28-day mortality was $15 \%$, 84-day mortality was $24 \%$, and 5 -year mortality was $56 \%$ with a further increase of mortality in cirrhotic patients [7]. Another study from the United Kingdom estimated a 5-year survival of $31.8 \%$ after index hospitalization with severe $\mathrm{AH}$. Abstinence from alcohol was the only independent predictor of longterm survival [8].

While mild AH usually enjoys a good prognosis with alcohol abstinence only, severe $\mathrm{AH}$ is associated with high mortality rates and requires additional treatment.

\footnotetext{
*Correspondence: Erik.f.rahimi@uth.tmc.edu

Division of Gastroenterology, Hepatology and Nutrition, University of Texas Medical School at Houston, 6431 Fannin Street, MSB 4.234, Houston, TX 77030, USA
}

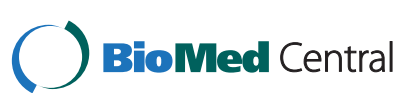

(c) 2015 Rahimi and Pan. This is an Open Access article distributed under the terms of the Creative Commons Attribution License (http://creativecommons.org/licenses/by/4.0), which permits unrestricted use, distribution, and reproduction in any medium, provided the original work is properly credited. The Creative Commons Public Domain Dedication waiver (http:// creativecommons.org/publicdomain/zero/1.0/) applies to the data made available in this article, unless otherwise stated.

\section{Maddrey discriminant function}

The American Association for the Study of Liver Diseases (AASLD) practice guidelines recommend that patients suspected of having AH should have their outcome risk stratified using the discriminant function (DF), along with other available clinical data [2]. In a placebo-controlled study assessing benefits of prednisolone in $\mathrm{AH}$, Maddrey et al. in 1978 first yielded the DF based on prothrombin time (PT) and serum bilirubin concentration that identified patients with a significant risk for early mortality [12]. This was later modified in 1989 to the modified DF (or $\mathrm{mDF}$ ) $=4.6$ (patient's PT in seconds- control PT in seconds) + total bilirubin $(\mathrm{mg} / \mathrm{dL})$ that is used today. Those patients with $\mathrm{mDF}>32$ were considered to have severe $\mathrm{AH}$. Patients with an elevated $\mathrm{mDF}$ and/or with encephalopathy that received corticosteroid therapy, showed a 28-day mortality of $6 \%$ in the treatment group compared to $35 \%$ in

Corticosteroids and pentoxifylline are the main pharmacological treatment options that have shown to improve short-term survival, although the overall outcomes are Estimating prognosis and identifying those who will need treatment is therefore extremely important. Progmodels are developed by combining two or more tially predict clinical outcomes [10]. Non-invasive scoring ing such as liver biopsies can lead to increased morbidity [11]. In this article, we review several scoring systems that are available to assess severity and prognosis of $\mathrm{AH}$. 
the placebo group [13]. On retrospective analysis, DF is a valid index of severity of disease identifying those at $50 \%$ risk of death within 2 months [14]. DF has an inadequate specificity of $<40 \%-62 \%$ and a sensitivity of $67 \%-100 \%$ for short-term (30 day) mortality $[15,16]$. The inaccuracy in using DF may be accounted for the cut-off point of 32, and had been the basis of debate on differing effectiveness of corticosteroid treatment. Higher cut-off values have been proposed such as cut-offs of $33,37,41,42$, or 44 to increase the specificity [15-18]. However, DF scores less than 32 have also been shown to be associated with a relatively high 28-day mortality of $16.7 \%$. DF was shown to have a poor diagnostic performance since it can only predict mortality or survival $66.6 \%$ of the time [15]. A drawback of using DF is the variable results of PT across different laboratories. The PT test relies on thromboplastin, a variable reagent, making inter-laboratory results vary greatly [19]. Due to the aforementioned limitations of DF, several other prognostic models as listed below have therefore been developed.

\section{Model for end-stage liver disease (MELD) score}

The MELD score is calculated using bilirubin, creatinine and international normalized ratio (INR) levels. The objective parameters were originally used to predict early death following elective transjugular intrahepatic portosystemic shunts (TIPS) [20]. The use of objective and reproducible data was subsequently shown to be a reliable measure of short-term mortality risk in patients with end-stage liver disease independent of complications of portal hypertension, and is used as a disease severity index to determine organ allocation priorities [21]. Several studies have used the MELD score to assess disease severity in AH. In a study by Sheth et al [18], the MELD score had a similar performance as the DF in predicting mortality at 30 days. The sensitivity and specificity in predicting 30-day mortality was $86 \%$ and $82 \%$, respectively, for MELD scores $>11$ compared to $86 \%$ and $48 \%$, respectively, when DF was greater than 32 . The utility of predicting mortality using MELD score represented as area under the curve (AUC) was 0.82 (95\% confidence intervals (CI): $0.65-0.98)$, and AUC of DF was 0.86 (95\% CI: 0.70-1.00). Sheth et al. therefore suggested that treatment for $\mathrm{AH}$ should be considered when MELD score is greater than 11.

Other studies have suggested higher cut-offs for MELD scores for predicting mortality. In a retrospective study, Srikureja et al [22] reported that MELD score is better than DF in predicting in-hospital mortality. An admission MELD score $\geq 18$ showed a sensitivity and specificity of $85 \%$ and $84 \%$, respectively, for predicting in-hospital mortality. First week MELD score $\geq 20$ had a $91 \%$ sensitivity and $85 \%$ specificity, and first week change of MELD score $\geq 2$ points had a $80 \%$ sensitivity and $75 \%$ specificity for predicting in-hospital mortality.
Dunn et al. [17], in a retrospective review of 73 patients showed that MELD score was the only independent predictor of mortality in patients with AH. MELD was shown to be comparable to DF in predicting 30-day and 90-day mortality. A MELD score of 21 in the study was shown to have a sensitivity of $75 \%$ and a specificity of $75 \%$ to predict mortality with an estimated 90-day mortality of $20 \%$ in AH. Another study reported a 30-day and 90-day mortality rates of $5.9 \%$ and $14.7 \%$, respectively in patients with AH. MELD score $>30.5$ had an excellent performance in predicting 30-day mortality with a sensitivity of $100 \%$ and specificity $94 \%$ (AUC 0.969). Furthermore, MELD score $>19$ only had a fair performance in predicting 90-day mortality with a sensitivity of $60 \%$ and a specificity of $60 \%$ (AUC 0.762) [23].

In a retrospective study of 26 patients, Vaa et al [24] compared MELD and MELD-Na in predicting 180-day mortality in patients with AH. MELD-Na is a modified MELD score that includes serum sodium $(\mathrm{Na})$ which has been shown to improve prediction of death in cirrhotic patients. In the study, MELD-Na was a better predictor of 180-day mortality than MELD in patients with ascites. A MELD score of 27 and a MELD-Na score of 28 in patients without ascites had a sensitivity/specificity of $76.5 \% / 64.9 \%$ and $87.5 \% / 52.5 \%$, respectively. After adjustment for MELD in AH patients without ascites, serum Na, specifically hyponatremia, was not a significant predictor of mortality $(p=0.83)$. However, in patients with ascites a MELD score of 29 and a MELD-Na score of 34 had sensitivity/specificity of $85.7 \% / 31.0 \%$ and $83.3 \% / 16.7 \%$, respectively. In AH patients with ascites, MELD-Na had better predictability of 180-day mortality compared to MELD (odds ratio (OR), 2.27 for every 1-point increase in MELD-Na; $95 \%$ CI: $1.22-36.68 ; p=0.008$ versus OR, 1.37 for every 1-point increase in MELD; 95 \% CI: 1.07-2.12; $p=0.006$ ).

Overall MELD score is a good predictor for mortality in patients with $\mathrm{AH}$. According to therapeutic algorithms, an initial MELD score $\geq 18$ and increasing serial MELD scores over time are considered high risk for mortality and should be used in guiding initiation of therapeutic intervention [2].

\section{Glasgow alcoholic hepatitis score (GAHS)}

In 2005, Forrest et al. [25] used 5 variables including age, blood urea, peripheral blood leukocyte count, serum bilirubin, and PT, expressed as a ratio of the control value to develop a new prognostic scoring system for $\mathrm{AH}$. Values obtained ranged from 5 to 12 , separated into those with value $<9$ or $\geq 9$ points. Day 28 survival in patients with a day 1 GAHS score of $<9$ was $87 \%$ compared to $46 \%$ in those with GAHS $\geq 9$. Based on the validation dataset, accuracy of GAHS day 1 and 7 data had a better prediction of 28 day and 84 day outcome than mDF. Accuracy of GAHS day 1 and 7 in predicting 
28-day mortality was $67 \%$ and $75 \%$, versus mDF accuracy of $48 \%$ and $56 \% ; p=0.0016$ and $p=0.0038$, respectively. Accuracy of GAHS day 1 and 7 in predicting 84-day mortality was $71 \%$ and $75 \%$, versus mDF accuracy of $57 \%$ and $62 \% ; p=0.0179$ and $p=0.0477$, respectively. In a subsequent study in 2007 by Forrest et al. [26], patients with a $\mathrm{mDF} \geq 32$ and a GAHS $<9$ did not benefit from treatment with corticosteroids. However corticosteroids therapy was associated with better survival in those with both GAHS $\geq 9$ and $\mathrm{mDF} \geq 32$ compared to no treatment (28-day survival $78 \%$ vs. $52 \%, p=0.002$ ). Day 84 survival was $59 \%$ and $38 \%(p=0.02)$ in those treated and not treated with steroids, respectively. In a recent study, Lafferty et al. [27] showed that patients receiving corticosteroids with a GAHS $\geq 9$, irrespective of $\mathrm{mDF}$, had a 90-day survival of $58 \%$ compared to $30 \%$ in those not receiving treatment. The sensitivity and specificity of 90-day outcome for GAHS assessment on admission was $67 \%$ and $78 \%$, respectively.

\section{Age, serum bilirubin, INR, and serum creatinine (ABIC) score}

In 2008, Dominguez et al. [28] built a predictive score from multivariate analysis of variables identified during admission. The resulting score: Age, serum Bilirubin, INR, and serum Creatinine $(\mathrm{ABIC})$ score $=($ age $\times 0.1)+($ serum bilirubin $\times 0.08)+($ serum creatinine $\times 0.3)+($ INR $\times 0.8)$ was validated in an independent prospective cohort. Using a cutoff value of 6.71 and 9 the score identified patients with $\mathrm{AH}$ that have a low (100\% survival), intermediate (70\% survival), and high risk ( $25 \%$ survival) of death at 90 days. The sensitivity and specificity was $100 \%$ and $50 \%$ for the cut-off of 6.71 , respectively, and $70 \%$ and $33 \%$, respectively, for a cut-off of 9 . In a retrospective study evaluating 9 different scoring systems, the sensitivity and specificity of the ABIC score for 30-day mortality were $100 \%$ and $20 \%$, respectively, for a cut-off of 6.71 , and $60 \%$ and $80 \%$, respectively, for a cut-off of 9. A higher ABIC cut-off of 9.5 compared to original cut-off of 9 resulted in an increased specificity of $90 \%$ vs. $80 \%$ and $95 \%$ vs. $84 \%$ for a 30 - and 90-day outcome respectively [16]. When comparing the 90-day mortality predictive accuracy of the ABIC score with MELD, mDF, and GAHS, the ABIC score was the best independent predictor of 90-day mortality (hazard ratio (HR) 2.78, $95 \%$ CI 1.90-4.09, $p=0.0001$ ). The ABIC score was also assessed to determine 1-year mortality, which could be used for liver transplantation assignment. The ABIC score was the only independent predictor of 1-year mortality (HR: 2.49, 95 \% CI 1.77-3.52, $p=0.0001)$, when compared to other prognostic models. Analysis of the subgroup of patients treated with steroids showed that the greatest response was in the group with intermediate $\mathrm{ABIC}$ score (between 6.71-8.99) compared to those with either low ABIC
$(<6.71)$ or high $\mathrm{ABIC}$ scores $(>9)$. In patients treated with steroids, the ABIC score at 7 days has a better accuracy than the Lille model in predicting mortality at 6 months [28].

\section{Lille model}

In 2007, Louvet et al. [29] generated a prognostic model, the Lille model, to identify "non-responders" to corticosteroid therapy in patients with severe AH. The model combined six objective variables (age, renal insufficiency, albumin, PT, bilirubin, and evolution of bilirubin at day 7) which were highly predictive of death at 6 months in patients treated with corticosteroids $(\mathrm{p}<0.000001)$. The Lille formula is available online at http://www.lillemodel.com or see formula in Table 1 . A cut-off value of 0.45 was determined to be the best identifier of patients at high risk of death, with a sensitivity and specificity of $81 \%$ and $76 \%$, respectively in the validation cohort, and $76 \%$ and $85 \%$, respectively on overall patients. Patients with a Lille score $\geq 0.45$ had a significant decrease in 6-month survival compared to patients with a Lille score $<0.45$ (25\% versus $85 \%$; $<0.0001$ ). Thus, $40 \%$ of patients receiving steroids can be identified to have a poor prognosis using the Lille model. Patients receiving corticosteroids after 7 days with a score $\geq 0.45$ may be futile and alternative treatments should be considered. In a prospective study regarding infection in AH patients, only the Lille model (OR, 11.14; $95 \%$ CI: 3.2-39.2, $p=0.0002)$ independently predicted infection upon steroids use in multivariate analysis. Responders (Lille model $<0.45$ ) to steroids that developed infection had lower survival compared to responders that did not develop infection: $51 \%$ vs $94 \%$, respectively $(p=0.000001)$. Non-responders (Lille model $\geq 0.45$ ) that developed infection had similar survival to nonresponders that did not develop infection: $42 \%$ vs $52 \%$, respectively $(p=0.5)$. Thus adjuvant antibiotic therapy to corticosteroids in the setting of severe $\mathrm{AH}$ may improve survival mainly in responders [30].

\section{Beclere model}

The Beclere model was initially formulated by Poynard et al. [31] in 1994 to determine survival in alcoholic cirrhosis. The final model has four variables: age, encephalopathy, serum bilirubin, and serum albumin to obtain a risk score $(\mathrm{R})$ for each patient using the formula $\mathrm{R}=(0.0484 \times$ [Age in Years $]+0.469 \times$ [encephalopathy $]+$ $0.537 \times \log _{e}$ [Bilirubin in $\left.\mu \mathrm{mol} / \mathrm{L}\right]-0.052 \times$ [Albumin in $\mathrm{g} / \mathrm{L}]$. This model was then used by Mathurin et al. [32] as a simulated prognostic model for a control group in a study aimed to examine prognostic factors and long-term survival in $\mathrm{AH}$ patients receiving corticosteroids. There was no difference in 1 and 2 year survival in the observed placebo-randomized group and the simulated control group using the Beclere model. In a multivariate analysis, 
Table 1 Prognostic scoring formulas to determine severity of acute alcoholic hepatitis

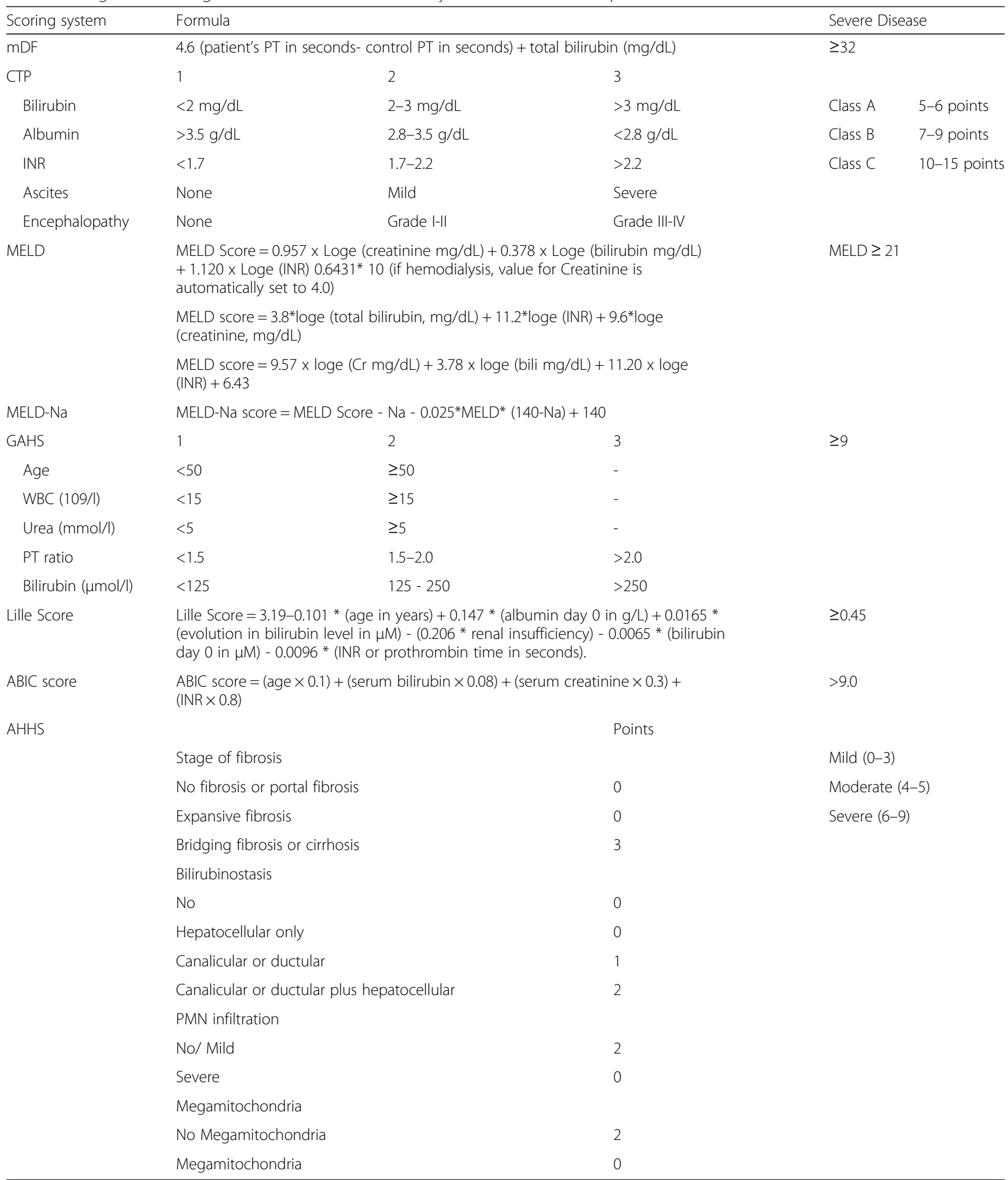

mDF modified Discriminant Function, CTP Child Turcotte Pugh, MELD Model for End-stage Liver Disease, GAHS Glasgow Alcoholic Hepatitis Score, ABIC Age serum Bilirubin INR and serum Creatinine, AHHS Alcoholic Hepatitis Histologic Score

the corticosteroid effect $(\mathrm{p}<0.02)$ and the Beclere model risk score $(p=0.0003)$ had independent prognostic value for survival. Survival was significantly better in the treated groups compared to the non-treated groups. The prednisolone - randomized group had $69 \%$ survival $(95 \% \mathrm{CI}$ : $57 \%-81 \%)$ and $71 \%(95 \% \mathrm{CI}: 55 \%-87 \%)$ in the 
prednisolone-open group, compared to $41 \%$ (95\% CI: $23 \%-59 \% ; p=0.01)$ in the placebo-randomized group and $50 \%(95 \% \mathrm{CI}: 37 \%-63 \% ; p=0.05)$ in the simulated control group. However, subsequent studies in $\mathrm{AH}$ patients did not further use this model for prognostic scoring.

\section{Alcoholic hepatitis histologic score (AHHS)}

The need for liver biopsy is controversial in diagnosing alcoholic hepatitis patients as the presence of ascites and coagulopathy may require a transjugular approach, which may not be readily available [33]. Altamirano et al. [34] developed a histologic scoring system based on liver biopsy findings to predict short-term (90-day) mortality in $\mathrm{AH}$ patients. The AHHS was initially developed from $121 \mathrm{pa}-$ tients admitted to a single center in Spain, and subsequently tested and validated in another set of 205 patients from 5 academic centers in the United States and Europe. After multivariate analysis, four independent histologic features were combined in the final score: fibrosis stage $(0-3)$ which was separated as bridging fibrosis or cirrhosis giving a score of +3 versus a score of 0 for absence of these features; bilirubinostasis $(0-2)$ was divided into a score of 0 for absence or hepatocellular only, +1 for canalicular or ductular, and +2 for canalicular or ductular plus hepatocellular; polymorphonuclear (PMN) infiltration (0-2) was described as "mild" PMN (score +2 ) when usually $<15$ PMN per focus were found around a hepatocyte and "marked" PMNs were easily recognized at low magnification and many PMNs found around damaged hepatocytes (with ballooning or Mallory-Denk bodies); and megamitochondria $(0-2)$ where none seen was +2 , and seen was 0 , for a total of 9 points. Marked PMN infiltration and megamitochondria were independently associated with a favorable outcome. AHHS cut-off score categorized patients as low 0-3 ( $97 \%$ survival), intermediate 4-5 (81 \% survival), and high risk 6-9 (49 \% survival) of death. When combing the AHHS with analytical scoring systems, the AHHS was able to refine the prognostic stratification of those with a MELD score <21 (low risk group). In patients with a MELD score $<21$ (low risk group) the AHHS was able to define 2 subgroups with different 90 -day survival using a cut-off of 5 points ( $94 \%$ vs $72 \% ; p=0.001)$. The differences in the MELD with an AHHS $<5$ points and those with an AHHS $\geq 5$ points $(16 \pm 8$ vs $23 \pm 9$ points of MELD, respectively; $\mathrm{p}<0.0001$ ) suggest that analytic parameters in the MELD score, such as bilirubin, are reflected in severity of histologic abnormalities. This would, therefore, modify stratification of severity from a low to high risk group, and change treatment management in these patients.

\section{Child-Turcotte-Pugh (CTP) score}

The CTP is based on 5 variables including ascites, encephalopathy, serum bilirubin, albumin and PT, which the latter was modified in 1973 by Pugh et al. from the original use of nutritional status in the Child-Turcotte criteria. Each variable has a score of 1 to 3, and patients are classified as class A (best), B (moderate), or C (worse) to determine prognosis, originally for cirrhotic patients undergoing surgery $[35,36]$. The limitations to the scoring system include subjectivity in ascites and encephalopathy grading, variable PT results, and a "ceiling" and "floor" effect for arbitrary cut-off points with bilirubin and albumin, respectively [37]. The use of CTP score in AH is not widely used. In 2004, Said et al [38] reported that CTP compared to the MELD score had similar predictive abilities for 3- and 6-month mortality in $\mathrm{AH}$ patients, with a c-statistics of $0.85(0.75-0.95)$ and 0.81 (0.70-0.92), respectively. Srikureja et al. [22] retrospectively compared MELD, CTP, and DF scores as predictive models to assess in-hospital mortality in AH patients. CTP score was independently associated with mortality on admission with an AUC of 0.87 (95\% CI: 0.81-0.94). However, this was lost with the first week change in CTP score (AUC 0.57; $95 \%$ CI: 0.43-0.70) compared to first week change in MELD score (AUC 0.85; $95 \%$ CI: 0.76-0.94; $p=0.0004$ ).

\section{TMA and pentane (TAP) score}

A recent study by Hanouneh et al. [39] identified novel breath biomarkers in patients with AH. Six compounds including 2-propanol, acetaldehyde, acetone, ethanol, pentane, and trimethylamine (TMA) were initially shown to be increased in patients with liver disease compared with healthy control subjects. TMA, acetone and pentane were significantly higher in AH compared to those with acute decompensation or control subjects (for all, $\mathrm{p}<0.001$ ). After accounting for MELD score only the associations between TMA and pentane (TAP) to AH remained significant (TMA, $\mathrm{p}<0.001$; pentane, $p=0.004$ ). Combining pentane and TMA levels in the breath was found to have an excellent prediction accuracy to diagnose $\mathrm{AH}$ (AUC 0.92). A model, named the TAP score, was then developed using the logistic regression (lr) function of the two variables $(\mathrm{l} r=-3.71+[0.34 * \mathrm{TMA}]-[0.087 *$ pentane $])$, and the following derived formula, TAP score $=100 \times(\exp [\mathrm{lr}] /$ $1+\exp$ [lr] was used. TAP scores of $\geq 36$ identified patients with $\mathrm{AH}$ with $90 \%$ sensitivity and $80 \%$ specificity. There correlation of the so called breathprint and severity of liver disease was only moderate as presented by MELD score $(\mathrm{r}=0.38 ; 95 \% \mathrm{CI}, 0.07-0.69 ; p=0.18)$. Larger studies are needed to further validate these results, as only a small group of 40 patients with $\mathrm{AH}$ were assessed.

\section{Combining static and dynamic models}

Louvet et al. [40] evaluated the prognostic value of combining static models for AH, such as mDF, MELD score, and ABIC score with dynamic models, such as the Lille score. This joint effect model was able to predict outcome 
of survival after 2 and 6 months significantly better than either the static or dynamic models alone $(\mathrm{p}<0.01)$. The MELD + Lille combination model predicted survival better than the $\mathrm{mDF}+$ Lille or $\mathrm{ABIC}+$ Lille models. Using the joint effect model of MELD + Lille score, a hypothetical patient with a MELD score of 21 and Lille score at 0.45 had a $15.3 \%$ and $23.7 \%$ mortality rate at 2 and 6 months, respectively. The overall predicted mortality at 6 months in the MELD + Lille model with a MELD score of 15-45 was between $8.5 \%-49.7 \%$ in a complete responder (Lille score, 0.16), and from 16.4 \%-75.2\% in a non-responder (Lille score, 0.45). The use of the joint effect models has a better prediction of mortality in $\mathrm{AH}$ patients. This models can also identify high risk of death in patients previously classified as responders, and intermediate risk of death in previous classified non-responders.

\section{Further indicators of prognosis in alcoholic hepatitis Acute kidney Injury (AKI)}

AKI, as per the AKIN (Acute Kidney Injury Network) criteria, is defined as an abrupt reduction (within $48 \mathrm{~h}$ ) in kidney function that results in an absolute increase of at least $0.3 \mathrm{mg} / \mathrm{dL}$ (or a $50 \%$ increase) in serum levels of creatinine from baseline [41]. In a retrospective study by Altamirano et al [42], AKI was shown to markedly influence 90 day mortality in patients with $\mathrm{AH}$ versus without AH $(65 \%$ vs. $7 \%, \mathrm{p}<0.0001)$. The most accurate predictors of AKI were the presence of systemic inflammatory response syndrome, serum bilirubin (especially $>16 \mathrm{mg} / \mathrm{dL}$ ), and elevated INR $>1.7$ in patients with AH.

\section{Change in bilirubin}

Similar to the Lille score, early change in bilirubin levels (ECBL), defined as bilirubin level at 7 days lower than bilirubin level on the first day of treatment of steroids was shown to be an important prognostic factor in $\mathrm{AH}$ patients. Mathurin et al [43] reported that $95 \%$ of patients with ECBL continued to have improved liver function during treatment. Six-month survival in patients with ECBL was $82.8 \pm 3.3 \%$ versus $23 \pm 5.8 \%(\mathrm{p}<0.0001)$ in the non-ECBL group. Another study by Morris and Forrest [44] identified steroid responders, defined as a $25 \%$ fall in serum bilirubin after 6-9 days of treatment had a better survival than non-responders. Non-responders were found to have 28-day and 56-day mortality of $36.8 \%$ and $57.9 \%$, respectively, versus responders with $0 \%(p=0.0148)$ and $11.1 \%(p=0.0084)$, respectively. The above studies, therefore, suggest stopping steroids in non-responders.

\section{Gastrointestinal (GI) bleed}

In a recent study, Rudler et al. [45] compared the mortality risk in $\mathrm{AH}$ patients who had concomitant
GI bleeding (AH-GIB+) with $\mathrm{AH}$ patients without GI bleeding (AH-GIB-). There was no difference in 1, 3, and 6-month probability of survival in $\mathrm{AH}-\mathrm{GIB}+$ versus $\mathrm{AH}$ GIB- groups $(87.9 \pm 4 \%$ vs $84.1 \pm 5 \%, p=0.56 ; 79.2 \pm 5 \%$ vs $71.1 \pm 7 \%, p=0.24$; and $73.9 \pm 6 \%$ vs $69.9 \pm 7 \%$, $p=0.49$, respectively). There was also no difference in response to therapy between the two groups as well.

\section{Protein-Calorie malnutrition}

Malnutrition, to some degree is found in every $\mathrm{AH}$ patient whether they do or do not have concomitant cirrhosis [46]. Mendenhall et al. [47] found that the severity of protein energy malnutrition (PEM) correlated with prognosis of $\mathrm{AH}$ patients. A moderate PEM of $60 \%-79 \%$ of normal was associated with a $29 \%$ 6-month mortality, and severe malnutrition (PEM score $<60 \%)$ correlated with a $45 \%$ mortality in 6 months. Patients with moderate malnutrition and adequate caloric intake $(>2,500 \mathrm{kcal} /$ day) that were given oxandrolone (an anabolic steroid) had a reduced 6-month mortality of $4 \%$ versus $28 \%$ in the placebo group $(p=0.002)$. These findings were not seen in cases of severe malnutrition [48]. In a randomized control trial, $\mathrm{AH}$ patients received either prednisone or total enteral nutrition (TEN) of 2,000 kcal/day for 28 days [49]. Short term (28-day period) mortality was $25 \%$ and $31 \%$ in the steroid group and TEN group, respectively. One-year survival probability was $39 \%$ with steroids and $62 \%$ with TEN. Thus there may be a synergistic effect in using steroid and TEN in AH treatment. In a meta-analysis, Antar et al. [50] pooled 7 randomized control trials that compared nutritional supplementation plus a normal hospital diet versus diet alone. There was no statistical difference in mortality between the two groups (OR, 0.80; 95 \% CI: 0.42-1.52), although there was a trend toward survival benefit with supplemental nutrition and significant improvement in encephalopathy (OR, 0.24; 95 \% CI: 0.06-0.93). Thus nutritional supplement is still regarded as beneficial.

\section{Conclusion}

The available scoring systems are useful in both prognostic stratification and selection of candidates for appropriate therapy. Limitations of the scoring systems include differing cut-offs to meet the best accuracy, clinical and laboratory parameters that may differ, and that one scoring system may be insufficient to determine some patients with severe $\mathrm{AH}$. Combining more than one scoring system, e.g. DF and Lille score, MELD and AHHS, or static and dynamic models may better define severe $\mathrm{AH}$ with greater accuracy. Furthermore, determining responders versus non-responders to therapy is important to avoid untoward adverse events associated with treatment. 


\section{Abbreviations}

AH: Alcoholic hepatitis; AST: Serum aspartate aminotransferase; ALT: Alanine aminotransferase; AASLD: American Association for the Study of Liver Diseases; DF: Discriminant function; PT: Prothrombin time; mDF: Modified DF; MELD: Model for End-Stage Liver Disease; INR: International normalized ratio; TIPS: Transjugular intrahepatic portosystemic shunts; AUC: Area under the curve; Cl: Confidence intervals; OR: Odds ratio; GAHS: Glasgow Alcoholic Hepatitis Score; ABIC: Age serum Bilirubin INR and serum Creatinine; HR: Hazard ratio; AHHS: Alcoholic Hepatitis Histologic Score; PMN: Polymorphonuclear; CTP: Child-Turcotte-Pugh; AKI: Acute Kidney Injury; AKIN: Acute Kidney Injury Network; ECBL: Early change in bilirubin levels; GI: Gastrointestinal; PEM: Protein energy malnutrition; TEN: Total enteral nutrition.

\section{Competing interests}

All authors disclose no conflicts and no competing interests.

\section{Authors' contributions}

ER: Drafting of the manuscript; critical revision of the manuscript for important intellectual content. J-JP: Drafting of the manuscript; critical revision of the manuscript for important intellectual content. Both authors read and approved the final manuscript.

\section{Received: 9 June 2015 Accepted: 10 July 2015}

\section{Published online: 21 July 2015}

\section{References}

1. Liangpunsakul S. Clinical characteristics and mortality of hospitalized alcoholic hepatitis patients in the United States. J Clin Gastroenterol. 2011;45(8):714-9.

2. O'Shea RS, Dasarathy S, McCullough AJ. Diseases PGCotAAftSoL, Gastroenterology PPCotACo. Alcoholic liver disease. Hepatology. 2010;51(1):307-28.

3. Lucey MR, Mathurin P, Morgan TR. Alcoholic hepatitis. N Engl J Med 2009;360(26):2758-69.

4. Ishak KG, Zimmerman HJ, Ray MB. Alcoholic liver disease: pathologic, pathogenetic and clinical aspects. Alcohol Clin Exp Res. 1991:15(1):45-66

5. Cohen JA, Kaplan MM. The SGOT/SGPT ratio-an indicator of alcoholic liver disease. Dig Dis Sci. 1979;24(11):835-8.

6. Yang AL, Vadhavkar S, Singh G, Omary MB. Epidemiology of alcohol-related liver and pancreatic disease in the United States. Arch Intern Med. 2008;168(6):649-56.

7. Sandahl TD, Jepsen $\mathrm{P}$, Thomsen $\mathrm{KL}$, Vilstrup $\mathrm{H}$. Incidence and mortality of alcoholic hepatitis in Denmark 1999-2008: a nationwide population based cohort study. J Hepatol. 2011;54(4):760-4.

8. Potts JR, Goubet $S_{1}$, Heneghan MA, Verma S. Determinants of long-term outcome in severe alcoholic hepatitis. Aliment Pharmacol Ther. 2013;38(6):584-95.

9. Chayanupatkul M, Liangpunsakul S. Alcoholic hepatitis: a comprehensive review of pathogenesis and treatment. World J Gastroenterol. 2014;20(20):6279-86

10. Perel $P$, Edwards $P$, Wentz $R$, Roberts I. Systematic review of prognostic models in traumatic brain injury. BMC Med Inform Decis Mak. 2006;6:38.

11. Liver EAftSo. EASL clinical practical guidelines: management of alcoholic liver disease. J Hepatol. 2012;57(2):399-420.

12. Maddrey WC, Boitnott JK, Bedine MS, Weber FL, Mezey E, White RI. Corticosteroid therapy of alcoholic hepatitis. Gastroenterology. 1978;75(2):193-9.

13. Carithers RL, Herlong HF, Diehl AM, Shaw EW, Combes B, Fallon HJ, et al. Methylprednisolone therapy in patients with severe alcoholic hepatitis. A randomized multicenter trial. Ann Intern Med. 1989;110(9):685-90.

14. Ramond MJ, Poynard T, Rueff B, Mathurin P, Théodore C, Chaput JC, et al. A randomized trial of prednisolone in patients with severe alcoholic hepatitis. N Engl J Med. 1992;326(8):507-12.

15. Kulkarni K, Tran T, Medrano M, Yoffe B, Goodgame R. The role of the discriminant factor in the assessment and treatment of alcoholic hepatitis. J Clin Gastroenterol. 2004;38(5):453-9.

16. Papastergiou V, Tsochatzis EA, Pieri G, Thalassinos E, Dhar A, Bruno S, et al. Nine scoring models for short-term mortality in alcoholic hepatitis: crossvalidation in a biopsy-proven cohort. Aliment Pharmacol Ther. 2014;39(7):721-32.
17. Dunn W, Jamil LH, Brown LS, Wiesner RH, Kim WR, Menon KV, et al. MELD accurately predicts mortality in patients with alcoholic hepatitis. Hepatology. 2005;41(2):353-8.

18. Sheth M, Riggs M, Patel T. Utility of the Mayo End-Stage Liver Disease (MELD) score in assessing prognosis of patients with alcoholic hepatitis. BMC Gastroenterol. 2002;2:2.

19. Robert $A$, Chazouillères $O$. Prothrombin time in liver failure: time, ratio, activity percentage, or international normalized ratio? Hepatology. 1996;24(6):1392-4.

20. Malinchoc M, Kamath PS, Gordon FD, Peine CJ, Rank J, ter Borg PC. A model to predict poor survival in patients undergoing transjugular intrahepatic portosystemic shunts. Hepatology. 2000;31(4):864-71.

21. Kamath PS, Wiesner RH, Malinchoc M, Kremers W, Therneau TM, Kosberg CL, et al. A model to predict survival in patients with end-stage liver disease. Hepatology. 2001:33(2):464-70.

22. Srikureja W, Kyulo NL, Runyon BA, Hu KQ. MELD score is a better prognostic model than Child-Turcotte-Pugh score or Discriminant Function score in patients with alcoholic hepatitis. J Hepatol. 2005:42(5):700-6.

23. Soultati AS, Dourakis SP, Alexopoulou A, Deutsch M, Vasilieva L, Archimandritis AJ. Predicting utility of a model for end stage liver disease in alcoholic liver disease. World J Gastroenterol. 2006;12(25):4020-5.

24. Vaa BE, Asrani SK, Dunn W, Kamath PS, Shah VH. Influence of serum sodium on MELD-based survival prediction in alcoholic hepatitis. Mayo Clin Proc. 2011;86(1):37-42.

25. Forrest EH, Evans CD, Stewart S, Phillips M, Oo YH, McAvoy NC, et al. Analysis of factors predictive of mortality in alcoholic hepatitis and derivation and validation of the Glasgow alcoholic hepatitis score. Gut. 2005;54(8):1174-9.

26. Forrest EH, Morris AJ, Stewart S, Phillips M, Oo YH, Fisher NC, et al. The Glasgow alcoholic hepatitis score identifies patients who may benefit from corticosteroids. Gut. 2007:56(12):1743-6.

27. Lafferty $\mathrm{H}$, Stanley AJ, Forrest EH. The management of alcoholic hepatitis: a prospective comparison of scoring systems. Aliment Pharmacol Ther. 2013;38(6):603-10.

28. Dominguez M, Rincón D, Abraldes JG, Miquel R, Colmenero J, Bellot $P$, et al. A new scoring system for prognostic stratification of patients with alcoholic hepatitis. Am J Gastroenterol. 2008;103(11):2747-56.

29. Louvet A, Naveau S, Abdelnour M, Ramond MJ, Diaz E, Fartoux L, et al. The Lille model: a new tool for therapeutic strategy in patients with severe alcoholic hepatitis treated with steroids. Hepatology. 2007;45(6):1348-54.

30. Louvet A, Wartel F, Castel H, Dharancy S, Hollebecque A, Canva-Delcambre V, et al. Infection in patients with severe alcoholic hepatitis treated with steroids: early response to therapy is the key factor. Gastroenterology. 2009;137(2):541-8.

31. Poynard T, Barthelemy P, Fratte S, Boudjema K, Doffoel M, Vanlemmens C, et al. Evaluation of efficacy of liver transplantation in alcoholic cirrhosis by a case-control study and simulated controls. Lancet. 1994;344(8921):502-7.

32. Mathurin P, Duchatelle V, Ramond MJ, Degott C, Bedossa P, Erlinger S, et al. Survival and prognostic factors in patients with severe alcoholic hepatitis treated with prednisolone. Gastroenterology. 1996;110(6):1847-53.

33. Liangpunsakul S, Kleiner DE. The alcoholic hepatitis histologic score: structured prognostic biopsy evaluation comes to alcoholic hepatitis. Gastroenterology. 2014;146(5):1156-8.

34. Altamirano J, Miquel R, Katoonizadeh A, Abraldes JG, Duarte-Rojo A, Louvet A, et al. A histologic scoring system for prognosis of patients with alcoholic hepatitis. Gastroenterology. 2014;146(5):1231-1239.e1231-1236.

35. Child CG, Turcotte JG. Surgery and portal hypertension. Major Probl Clin Surg. 1964;1:1-85.

36. Pugh RN, Murray-Lyon IM, Dawson JL, Pietroni MC, Williams R. Transection of the oesophagus for bleeding oesophageal varices. Br J Surg. 1973;60(8):646-9.

37. Cholongitas E, Papatheodoridis GV, Vangeli M, Terreni N, Patch D, Burroughs AK. Systematic review: The model for end-stage liver disease-should it replace Child-Pugh's classification for assessing prognosis in cirrhosis? Aliment Pharmacol Ther. 2005;22(11-12):1079-89.

38. Said A, Williams J, Holden J, Remington P, Gangnon R, Musat A, et al. Model for end stage liver disease score predicts mortality across a broad spectrum of liver disease. J Hepatol. 2004;40(6):897-903.

39. Hanouneh IA, Zein NN, Cikach F, Dababneh L, Grove D, Alkhouri N, et al. The breathprints in patients with liver disease identify novel breath biomarkers in alcoholic hepatitis. Clin Gastroenterol Hepatol. 2014;12(3):516-23.

40. Louvet A, Labreuche J, Artru F, Boursier J, Kim DJ, O'Grady J, et al. Combining Data From Liver Disease Scoring Systems Better Predicts Outcomes of Patients With Alcoholic Hepatitis. Gastroenterology 2015. In Press. 
41. Mehta RL, Kellum JA, Shah SV, Molitoris BA, Ronco C, Warnock DG, et al. Acute Kidney Injury Network: report of an initiative to improve outcomes in acute kidney injury. Crit Care. 2007;11(2):R31.

42. Altamirano J, Fagundes C, Dominguez M, García E, Michelena J, Cárdenas A, et al. Acute kidney injury is an early predictor of mortality for patients with alcoholic hepatitis. Clin Gastroenterol Hepatol. 2012;10(1):65-71.e63.

43. Mathurin P, Abdelnour M, Ramond MJ, Carbonell N, Fartoux L, Serfaty L, et al. Early change in bilirubin levels is an important prognostic factor in severe alcoholic hepatitis treated with prednisolone. Hepatology. 2003;38(6):1363-9.

44. Morris JM, Forrest EH. Bilirubin response to corticosteroids in severe alcoholic hepatitis. Eur J Gastroenterol Hepatol. 2005;17(7):759-62.

45. Rudler M, Mouri S, Charlotte F, Lebray P, Capocci R, Benosman H, et al. Prognosis of treated severe alcoholic hepatitis in patients with gastrointestinal bleeding. J Hepatol. 2015;62(4):816-21.

46. Mendenhall CL, Anderson S, Weesner RE, Goldberg SJ, Crolic KA. Protein-calorie malnutrition associated with alcoholic hepatitis. Veterans Administration Cooperative Study Group on Alcoholic Hepatitis. Am J Med. 1984;76(2):211-22.

47. Mendenhall CL, Moritz TE, Roselle GA, Morgan TR, Nemchausky BA, Tamburro $\mathrm{CH}$, et al. A study of oral nutritional support with oxandrolone in malnourished patients with alcoholic hepatitis: results of a Department of Veterans Affairs cooperative study. Hepatology. 1993;17(4):564-76.

48. Mendenhall CL, Moritz TE, Roselle GA, Morgan TR, Nemchausky BA, Tamburro $\mathrm{CH}$, et al. Protein energy malnutrition in severe alcoholic hepatitis: diagnosis and response to treatment. The VA Cooperative Study Group \#275. JPEN J Parenter Enteral Nutr. 1995;19(4):258-65.

49. Cabré E, Rodríguez-Iglesias P, Caballería J, Quer JC, Sánchez-Lombraña $J \mathrm{~L}$, Parés A, et al. Short- and long-term outcome of severe alcoholinduced hepatitis treated with steroids or enteral nutrition: a multicenter randomized trial. Hepatology. 2000;32(1):36-42.

50. Antar $R$, Wong P, Ghali P. A meta-analysis of nutritional supplementation for management of hospitalized alcoholic hepatitis. Can I Gastroenterol. 2012;26(7):463-7.

\section{Submit your next manuscript to BioMed Central and take full advantage of:}

- Convenient online submission

- Thorough peer review

- No space constraints or color figure charges

- Immediate publication on acceptance

- Inclusion in PubMed, CAS, Scopus and Google Scholar

- Research which is freely available for redistribution 